\title{
Aspergilose e mucormicose - micoses sistêmicas de importância em COVID-19: Artigo de revisão
}

\author{
Aspergillosis and mucormycosis - systemic mycoses of importance in COVID-19: Review article \\ Aspergilosis y mucormicosis - micosis sistémicas de importancia en COVID-19: Artículo de revisión
}

Recebido: 11/06/2021 | Revisado: 18/06/2021 | Aceito: 22/06/2021 | Publicado: 26/06/2021

\author{
Ramiro Moreira Tavares \\ ORCID: https://orcid.org/0000-0002-1000-5468 \\ Hospital Universitário Júlio Bandeira, Brasil \\ E-mail: ramiromtavares@gmail.com \\ Cicero Emanoel Alves Leite \\ ORCID: https://orcid.org/0000-0001-8981-493X \\ Hospital Universitário Júlio Bandeira, Brasil \\ E-mail: emanoel.leite.ceal@gmail.com \\ Ravel Moreira Tavares \\ ORCID: https://orcid.org/0000-0002-4673-5032 \\ Programa Mais Médicos para o Brasil, Brasil \\ E-mail: ravel.cartola@hotmail.com \\ Patrícia Lopes Oliveira \\ ORCID: https://orcid.org/0000-0002-3319-3908 \\ Hospital Universitário Júlio Bandeira, Brasil \\ E-mail: patricia.oliveira.2@ebserh.gov.br \\ Ocilma Barros de Quental \\ ORCID: https://orcid.org/0000-0002-4075-2755 \\ Faculdade Santa Maria, Brasil \\ E-mail: ocilmaquental2011@hotmail.com
}

\begin{abstract}
Resumo
Introdução: A aspergilose invasiva e a mucormicose são infecções alarmantes em receptores de transplantes de órgãos sólidos e células hematopoiéticas. No contexto da pandemia causada pelo SARS-CoV2, têm-se evidenciados casos destas condições associadas a aumento de permanência hospitalar e desfechos sombrios. As infecções fúngicas invasivas representam entidades relevantes na atenção de pacientes graves afetados por este coronavírus. Objetivo: avaliar a importância da aspergilose e mucormicose em paciente acometidos por COVID-19. Metodologia: Trata-se de um estudo de revisão narrativa que se configura como estudo qualitativo e exploratório. Para tal, realizou-se análise de artigos publicados na base de dados PUBMED, assim como de livros impressos refrente à temática. Os termos utilizados para a pesquisa foram: "Aspergillosys", "Mucormycosis" e "Coronavirus Infections". Resultados: Aspergilose invasiva e mucormicose são complicações relacionadas com o aumento de mortalidade em pacientes com COVID-19. Conclusão: A aspergilose invasiva e a mucormicose são entidades com elevada mortalidade, representando efeito colateral que deve ser atentado precocemente em casos de deterioração de quadro clínico de indivíduo submetido a tratamento com corticosteroides e imunossupressores com COVID-19.
\end{abstract}

Palavras-chave: Aspergilose; Mucormicose; Infecções por Coronavirus.

\begin{abstract}
Introduction: Invasive aspergillosis and mucormycosis are alarming infections in solid organ and hematopoietic cell transplant recipients. In the context of the pandemic caused by SARS-CoV2, cases of these conditions associated with increased hospital stay and dismal outcomes have been evidenced. Invasive fungal infections represent relevant entities in the care of critically ill patients affected by this coronavirus. Objective: to evaluate the importance of aspergillosis and mucormycosis in patients affected by COVID-19. Methodology: This is a narrative review study that is configured as a qualitative and exploratory study. To this end, an analysis of articles published in the PUBMED database was carried out, as well as of printed books on the subject. The terms used for the research were: "Aspergillosys", "Mucormycosis" and "Coronavirus Infections". Results: Invasive aspergillosis and mucormycosis are complications related to increased mortality in patients with COVID-19. Conclusion: Invasive aspergillosis and mucormycosis are entities with high mortality, representing a side effect that should be addressed early in cases of deterioration in the clinical condition of an individual undergoing treatment with corticosteroids and immunosuppressants with COVID-19.
\end{abstract}

Keywords: Aspergillosis; Mucormycosis; Coronavirus infections. 


\begin{abstract}
Resumen
Introducción: La aspergilosis invasiva y la mucormicosis son infecciones alarmantes en receptores de trasplantes de órganos sólidos y células hematopoyéticas. En el contexto de la pandemia causada por el SARS-CoV2, se han evidenciado casos de estas afecciones asociadas con un aumento de la estadía hospitalaria y resultados desalentadores. Las infecciones fúngicas invasivas representan entidades relevantes en la atención de pacientes críticamente enfermos afectados por este coronavirus. Objetivo: evaluar la importancia de la aspergilosis y mucormicosis en pacientes afectados por COVID-19. Metodología: Se trata de un estudio de revisión narrativa que se configura como un estudio cualitativo y exploratorio. Para ello, se realizó un análisis de los artículos publicados en la base de datos PUBMED, así como de libros impresos sobre el tema. Los términos utilizados para la investigación fueron: "Aspergilosis", "Mucormicosis" e "Infecciones por coronavirus". Resultados: La aspergilosis invasiva y la mucormicosis son complicaciones relacionadas con el aumento de la mortalidad en pacientes con COVID-19. Conclusión: La aspergilosis invasiva y la mucormicosis son entidades con alta mortalidad, representando un efecto secundario que debe ser abordado precozmente en los casos de deterioro de la condición clínica de un individuo en tratamiento con corticoides e inmunosupresores con COVID-19.
\end{abstract}

Palabras clave: Aspergilosis; Mucormicosis; Infecciones por coronavirus.

\title{
1. Introdução
}

A aspergilose invasiva e a mucormicose são infecções alarmantes em receptores de transplantes de órgãos sólidos e células hematopoiéticas. No contexto da pandemia causada pelo SARS-CoV2, têm-se evidenciados casos destas condições associadas a aumento de permanência hospitalar e desfechos sombrios. As infecções fúngicas invasivas representam entidades relevantes na atenção de pacientes graves afetados por este coronavírus.

A COVID-19 é uma doença infecciosa causada por um quadro agudo gerado pelo SARS-CoV2 que foi identificado pela primeira vez em dezembro de 2019 em Wuhan, China, e atualmente está circulando em todo o mundo (Wang et al., 2020). A transmissão humano-humano se dá pelo contato com mãos contaminadas, aerossóis e secreções de pessoas infectadas, bem como fômites contaminados, sendo a via aerógena de extrema importância para a propagação do vírus (Corroza et al., 2020).

Uma vez inalado o SARS-CoV2, o processo infeccioso pode ter seu início. A incorporação do vírus às células respiratórias pneumócitos 2 ocorre quando o complexo proteína espicular S entra em contato com o receptor da enzima conversora da angiotensina II (ECA2) presente na membrana celular. A proteína S interage com a Proteinase Transmembrana Serina 2 (TMPRSS2), que também colabora decisivamente para a infecção. A ECA2 promove a fusão entre a superfície do vírus e a membrana plasmática, e a TMPRSS2 facilita, na sequência, a entrada do RNA na célula alvo. Após a inoculação do vírus na célula, o início dos sintomas se dá pela ativação da resposta imunológica por recrutamento celular e liberação de citocinas, quimiocinas e mediadores inflamatórios (tempestade de citocinas), que é ineficaz a contenção do vírus e produtora de dano tissular. A infecção pelo SARS-CoV pode causar dano aos linfócitos B e T e células NK, o que pode levar ao comprometimento do sistema imune. O processo de reposta pode causar lesão pulmonar grave podendo cursar com disfunção orgânica, inclusive com desfechos fatais (Neufeld, 2020).

Em paciente gravemente afetados pela COVID-19, com quadros inflamatórios mais intensos, é visto em estudos o desequilíbrio da microbiota por longo curso, sendo fator relevante para a emergência de fungos invasivos (Zuo et al., 2020). Fator este que é intensificado pela utilização de terapia antimicrobiana, iniciada por vezes precocemente em domicílio e mantida com espectros mais abrangentes em ambiente nosocomial, deixando o indivíduo a micoses por Candida sp, Aspergillus, Mucorales e outros fungos.

No tratamento da COVID-19, onde cada vez mais tem se visto a importância de drogas imunossupressoras, como corticosteroides e inibidores de IL-6, em pacientes graves com risco aumentado a desenvolver danos celulares e microtromboses, secundária a tempestade de citocinas, a ocorrência de micoses profundas associadas maior gravidade dos doentes.

Diante desse contexto, o presente estudo teve como objetivo avaliar a importância da aspergilose e mucormicose em paciente acometidos por COVID-19. 


\section{Metodologia}

Trata-se de um estudo de revisão narrativa que se configura como estudo qualitativo e exploratório com a finalidade de aprofundar a discussão da importância das mucomicoses associadas à COVID-19. De acordo com Köche (2011), o objetivo da pesquisa bibliográfica é o de conhecer e analisar as principais contribuições teóricas existentes sobre determinado tema ou problema. Para tal, realizou-se análise de artigos publicados na base de dados PUBMED, assim como de livros impressos refrente à temática.

Os termos utilizados para a pesquisa foram: "Aspergillosys", "Mucormycosis" e "Coronavirus Infections". O período considerado foi de artigos publicados até o mês de maio/2021 sem restrição quanto ao idioma de publicação. Inicialmente, título e resumo foram lidos para triar os de interesse para o presente estudo. Em seguinda, procedeu-se com a leitura do documento na íntegra para análise.

\section{Resultados e Discussão}

A COVID-19 é uma infecção grave com risco importante para desenvolvimento de desfechos trombembólicos em pacientes críticos, sendo idade avançada, comorbidades, gravidez, insuficiência cardíaca congestiva ou história pessoal de tromboembolismo fatores relacionados a aumento de mortalidade (Marinho et al., 2021), secundária a inflamação sistêmica, sendo a utilização de corticoterapia e imunobiológicos como base de suporte de doentes em terapia intensiva. A ocorrência de coinfecção por outros agentes vem sendo relatada, as micoses invasivas associadas podem potencializar o risco de desfechos indesejados.

Diante da inflamação robusta e a imunossupressão simultânea (sobretudo em pacientes com morbidades como o diabetes mellitus) evidente no manejo da COVID-19, acredita-se que a resposta imune desregulada fornece um hospedeiro propício ao desenvolvimento de infecções fúngicas (Mekonnen et al., 2021).

A associação de micoses sistêmicas, em especial a mucormicose (são descritos na literatura casos de associação das condições), e a aspergilose invasiva é observada em casos em todo mundo. Os fungos que causam estas doenças invasivas têm por características serem ubíquos, e assim como grande parte dos fungos geram condições graves em associação com condições que geram dano ao sistema imune relevante, podendo caracterizar-se como doenças oportunistas.

Mucormicose (anteriormente chamada zigomicose) é uma infecção oportunista, causadas por um fungo da classe Glomeromycetes, subfilo Mucoromycotina, ordem Mucorales, incluindo Rhizopus spp, Mucor spp, Lichtheimia (anteriormente Absidia), que residem no ambiente e podem infectar o homem na forma de esporos, que podem fazer doença rinocerebelar, pulmonar, gastrointestinal, disseminada, cutânea e cerebral (Jeffery-Smith et al., 2017). Os fungos atingem os vasos sanguíneos provocando trombose e infarto na região afetada. O diagnóstico depende do exame direto e histopatológico dos achados de biopsia de material necrótico, a cultura geralmente é negativa, diagnóstico este que geralmente é feito pós morte (Tavares \& Marinho, 2015).

A incidência da mucormicose varia de acordo com a condição de base do paciente, seu desfecho varia de acordo com a exposição, sítio de infecção e uso de terapia antimicrobiana. Estudos determinam que é uma doença predominantemente masculina, sendo a associação com diabetes e doença oncológica determinante, sendo a mortalidade geral da condição variando entre 36-44\% em países de língua inglesa (Roden et al., 2005).

Há relatos na literatura da condição evidenciada na faixa pediátrica, sobretudo em sua forma gástrica. A zigomicose é uma infecção com risco de vida em crianças e neonatos com diferentes padrões de envolvimento em indivíduos de diferentes idades. Trabalhos relacionam a gravidade da condição com prematuridade como principal fator na faixa neonatal $(<1$ mês $)$ sendo a mortalidade estimada em 64\% (maior que em adultos) (Roilides et al., 2009). 
Em adultos DM e doenças oncológicas são fortes fatores de risco isolados, e notavelmente, a combinação de terapia com esteroides para tratar SRAG pelo SRAS-CoV2 e DM pode aumentar a imunossupressão e a hiperglicemia, aumentando o risco de mucormicose em um indivíduo suscetível (Ahmadikia et al., 2021). O diabetes mellitus assume papel importante como fator de imnussupressão crônica aliada à terapia imunossupressora na ocorrência de infecção por fungos da ordem Mucorales (Mekonnen et al., 2021). Hiperglicemia, cetoacidose diabética e distúrbios metabólicos, e eletrolíticos, favorecem o crescimento fúngico e precisam de compensação em abordagem dos pacientes (Mekonnen et al., 2021).

$\mathrm{O}$ tratamento envolve remover cirurgicamente todos os tecidos mortos e infectados. Em alguns pacientes, isso pode resultar em deformidades a depender a apresentação (perda da mandíbula superior e ocular em apresentação rinocerebelar) (CDC, n.d.). A abordagem com antifúngicos se mostra relevante, Mucorales é intrínsecamente resistente a alguns antifúngicos, como fluconazol, voriconazol, fluocitosina e equinocandinas, sendo a anfotericina B a terapia de escolha, sobretudo em sua forma lipossomal, em razão de nefrotoxicidade (Marsh et al., 2018). Há relatos na literatura de terapia combinada de anfotericina B com posaconazol e isavuconazol têm mostrado resultados (Mekonnen et al., 2021), no entanto com resultados questionáveis.

O Aspergillus sp é um fungo filamentoso que pode causar doença em humanos, sendo o agente mais comum causador de doença fúngica invasiva no mundo com taxa de mortalidade superior a $80 \%$ a depender do centro (Bongomin et al., 2017). Ocorre em indivíduos imunodeprimidos, pode-se observar em pacientes neutropênicos, oncológicos e transplantados (Tavares \& Marinho, 2015). Destacam-se o A. fumigatus e A. negrii e outros.

A aspergilose pode se manifestar como doença superficial e profunda, tendo espectro vasto de apresentação, inclusive quadros alérgicos (reação orgânica a presença do fungo, geralmente de vias aéreas: sinusites, bronquites e quadros pulmonares alérgicos), doença crônica cavitaria e doença invasiva (Tavares \& Marinho, 2015).

A diminuição dos linfócitos e da função imunológica do hospedeiro pode ser uma das principais razões para as infecções pelo fungo (Neufeld, 2020). Os pacientes imunodeprimidos têm risco aumentado de desenvolvimento de forma disseminada e invasiva (Tavares \& Marinho, 2015). Da doença invasiva pode-se manifestar por diversas vias: angioinvasiva, não angioinvasiva, rinocerebelar, maxilar, cerebral e outras (Neufeld, 2020).

No início dos anos 2000, na epidemia de SARS-CoV na Ásia, e de MARS-CoV no Oriente Médio, foram descritos casos, em necropsias, de aspergilose, em pacientes que fizeram uso de corticoterapia. Casos de pacientes que desenvolveram infecção pulmonar invasiva por Asprgillus também foram descritos pós infecção pelo vírus Influenza A, dentre eles H1N1 e H3N2. Em período de pandemia pelo SARS-CoV2, tem-se visto uma associação da infecção por este fungo em doentes graves e inflamados, sobretudo em uso de terapia imunossupressora com corticosteroides, contribuindo para o aumento de mortalidade desta condição (H. Wang et al., 2003).

O diagnóstico de aspergilose invasiva se dá por achados clínicos e de imagem, em contexto de quadro pulmonar grave. Se observa incialmente a presença de febre que não responde aos antimicrobianos (Tavares \& Marinho, 2015). É uma doença que se caracteriza pela formação de escavação pleural e escavação progressiva, sendo a apresentação cavitária possível, simulando o diagnóstico de doença por Mycobacterium tuberculosis. A análise microbiológica de secreções com pesquisa do fungo por lavado broncoalveolar ou broncoscopia é de grande utilidade por meios de cultura específicos para o fungo, como também por métodos de biologia molecular (PCR) (Neufeld, 2020). A utilização de galactomana sérica ou pela sua mensuração em secreções de via aérea podem ser úteis no diagnóstico. No entanto devemos atentar para possíveis resultados falso-positivos em casos de outros fungos estarem presentes (p. ex: Fusarium sp. e Histoplasma capsulatum) (Martín-Rabadán et al., 2012). 
Estudos relacionam a ocorrência de Aspergilose invasiva duas vezes maior em indivíduos submetidos a intubação orotraqueal e com mais desfechos desfavoráveis a internação (Permpalung et al., 2021). Em estudo italiano, Bartoletti et al. (2020) relaciona a mortalidade de associação de COVID-19 com a micose invasiva em torno de 25\%.

A associação da micose com uso de terapia imunossupressora em contexto da COVID-19 vem sendo referida em publicações, sendo seu diagnóstico relacionado a utilização de inibidor de IL6, o tocilizumabe, que no contexto de sua utilização pode propiciar a Aspergilose invasiva em contexto de eliminação da via do IL6 responsável pela proteção contra o fungo (Lai \& Yu, 2021).

O tratamento da aspergilose invasiva se dá com o uso de antifúngicos, sendo a droga padrão ouro o Voriconazol, sendo o agente preferido em casos de doença grave, no entanto sua utilização pode cursar com cardiotoxicidade (prolongamento de intervalo QT), nefrotoxicidade, e interação com outras drogas por metabolismo via citocromo P450, sendo o isovoriconazol e o posaconazol, opções mais seguras devido perfil cardioprotetor em relação ao primeiro (Lai \& Yu, 2021). Casos graves e que não respondem a monoterapia com voriconazol há a recomendação de associação com equinocandinas, sobretudo a anidulofungina, esta associação também deve ser considerada na ocorrência de A. calidoustus e A. terréus, pois são espécies com altas concentrações inibitórias mínimas para numerosos antifúngicos (Marr et al., 2015). A anfotericina B desoxicolato, a anfotericina B lipossomal e anfotericina b complexo lipídico também são alternativas adicionais, mas esses agentes apresentam o risco de nefrotoxicidade e estão disponíveis apenas por via intravenosa (Berto \& Dalzochio, 2021).

$\mathrm{O}$ uso de associação de voriconazol e anfotericina se sustenta em casos de suspeita de coinfecção com fungo invasivo resistente, como o Mucorales, sendo neste contexto a terapia com Anfotericina B lipossomal a de escolha (Imhof et al., 2004).

\section{Considerações Finais}

O seguimento de pacientes graves com COVID-19 se faz complexo e a ocorrência de infecções por outros agentes associados é tema de extrema relevância, sendo as micoses invasivas responsáveis por aumento de tempo de hospitalização, custos hospitalares e vidas perdidas. A aspergilose invasiva e a mucormicose são entidades com elevada mortalidade, representando efeito colateral que deve ser atentado precocemente em casos de deterioração de quadro clínico de indivíduo submetido a tratamento com corticosteroides e imunossupressores com COVID-19. O diagnóstico precoce, a partir de aumento de critérios de exposição e o tratamento adequado em centros médicos são cruciais ao desfecho destes pacientes.

\section{Referências}

Ahmadikia, K., Jamal Hashemi, S., Khodavaisy, S., Ibrahim Getso, M., Alijani, N., Badali, H., Mirhendi, H., Salehi, M., Tabari, A., Mohammadi Ardehali, M., Kord, M., Roilides, E., \& Rezaie, S. (2021). The double-edged sword of systemic corticosteroid therapy in viral pneumonia: A case report and comparative review of influenza-associated mucormycosis versus COVID-19 associated mucormycosis. Mycoses, 00, 1-11. https://doi.org/10.1111/myc.13256

Bartoletti, M., Pascale, R., Cricca, M., Rinaldi, M., Maccaro, A., Bussini, L., Fornaro, G., Tonetti, T., Pizzilli, G., Francalanci, E., Giuntoli, L., Rubin, A., Moroni, A., Ambretti, S., Trapani, F., Vatamanu, O., Ranieri, V. M., Castelli, A., Baiocchi, M., PREDICO study group. (2020). Epidemiology of invasive pulmonary aspergillosis among COVID-19 intubated patients: a prospective study. Clinical Infectious Diseases: An Official Publication of the Infectious Diseases Society of America, ciaa1065. https://doi.org/10.1093/cid/ciaa1065

Berto, C., \& Dalzochio, T. (2021). Nephrotoxicity and fungal resistance associated with Amphotericin B. Saúde e Meio Ambiente: Revista Interdisciplinar, 10, 45-62. https://doi.org/10.24302/sma.v10.3136

Bongomin, F., Gago, S., Oladele, R. O., \& Denning, D. W. (2017). Global and Multi-National Prevalence of Fungal Diseases-Estimate Precision. Journal of Fungi (Basel, Switzerland), 3(4), 57. https://doi.org/10.3390/jof3040057

CDC. (n.d.). Symptoms of Mucormycosis | Mucormycosis | CDC. Centers for Disease Control and Prevention. https://www.cdc.gov/fungal/diseases/mucormycosis/symptoms.html

Corroza, M., Naranjo, L., Romero, A., Schmauck, T., \& Veja, E. (2020). COVID-19 Aspectos biológicos, clínicos y sociológicos: uma revisón. Nulliusin Verba Site, 1-25.

Imhof, A., Balajee, S. A., Fredricks, D. N., Englund, J. A., \& Marr, K. A. (2004). Breakthrough fungal infections in stem cell transplant recipients receiving 
voriconazole. Clinical Infectious Diseases: An Official Publication of the Infectious Diseases Society of America, 39(5), 743-746. https://doi.org/10.1086/423274

Jeffery-Smith, A., Taori, S. K., Schelenz, S., Jeffery, K., Johnson, E. M., Borman, A., Candida auris Incident Management Team, Manuel, R., \& Brown, C. S. (2017). Candida auris: a Review of the Literature. Clin Microbiol Rev, 31(1), e00029-17. https://doi.org/10.1128/CMR.00029-17

Köche, J. C. (2011). Fundamentos de metodologia científica: teoria da ciência e iniciação à pesquisa. Vozes

Lai, C. C., \& Yu, W. L. (2021). COVID-19 associated with pulmonary aspergillosis: A literature review. Journal of Microbiology, Immunology and Infection, 54(1), 46-53. https://doi.org/10.1016/j.jmii.2020.09.004

Marinho, V. R. D., Villarim, C. V. O., Silva, L. C. C. e, Silva, A. L. C. e, Araújo-Neto, I., Caldas, D. M. D. R., Rêgo, A. C. M., \& Araújo-Filho, I. (2021). Use of heparin in patients with severe COVID-19: What is the evidence? Research, Society and Development, 10(6), e28910615820. https://doi.org/10.33448/rsdv10i6.15820

Marr, K. A., Schlamm, H. T., Herbrecht, R., Rottinghaus, S. T., Bow, E. J., Cornely, O. A., Heinz, W. J., Jagannatha, S., Koh, L. P., Kontoyiannis, D. P., Lee, D. G., Nucci, M., Pappas, P. G., Slavin, M. A., Queiroz-Telles, F., Selleslag, D., Walsh, T. J., Wingard, J. R., \& Maertens, J. A. (2015). Combination antifungal therapy for invasive aspergillosis a randomized trial. Annals of Internal Medicine, 162(2), 81-89. https://doi.org/10.7326/M13-2508

Marsh, B. M., Rajasingham, R., Tawfic, S. H., \& Borofsky, M. S. (2018). Successful Conservative Management of Bilateral Renal Mucormycosis. Urology, 120, 2-5. https://doi.org/10.1016/j.urology.2018.03.010

Martín-Rabadán, P., Gijón, P., Fernández, R. A., Ballesteros, M., Anguita, J., \& Bouza, E. (2012). False-positive Aspergillus Antigenemia Due to Blood Product Conditioning Fluids. Clinical Infectious Diseases, 55(4), e22-e27. https://doi.org/10.1093/cid/cis493

Mekonnen, Z. K., Ashraf, D. C., Jankowski, T., Grob, S. R., Vagefi, M. R., Kersten, R. C., Simko, J. P., \& Winn, B. J. (2021). Acute Invasive Rhino-Orbital Mucormycosis in a Patient with COVID-19-Associated Acute Respiratory Distress Syndrome. Ophthalmic Plastic and Reconstructive Surgery, 37(2), E80. https://doi.org/10.1097/IOP.0000000000001889

Neufeld, P. M. (2020). A COVID-19 e o diagnóstico da aspergilose pulmonar invasiva. Rev. Bras. An. Clin., 52(2), 173-185. https://doi.org/10.21877/24483877.20200019

Permpalung, N., Chiang, T. P., Massie, A. B., Zhang, S. X., Avery, R. K., Nematollahi, S., Ostrander, D., Segev, D. L., \& Marr, K. A. (2021). COVID-19 Associated Pulmonary Aspergillosis in Mechanically Ventilated Patients. Clinical Infectious Diseases: An Official Publication of the Infectious Diseases Society of America, ciab223. https://doi.org/10.1093/cid/ciab223

Roden, M. M., Zaoutis, T. E., Buchanan, W. L., Knudsen, T. A., Sarkisova, T. A., Schaufele, R. L., Sein, M., Sein, T., Chiou, C. C., Chu, J. H., Kontoyiannis, D. P., \& Walsh, T. J. (2005). Epidemiology and Outcome of Zygomycosis: A Review of 929 Reported Cases. Clinical Infectious Diseases : An Official Publication of the Infectious Diseases Society of America, 41(5), 634-653. https://doi.org/10.1086/432579

Roilides, E., Zaoutis, T. E., \& Walsh, T. J. (2009). Invasive zygomycosis in neonates and children. Clinical Microbiology and Infection, 15(SUPPL. 5), 50-54. https://doi.org/10.1111/j.1469-0691.2009.02981.x

Tavares, W., \& Marinho, L. A. C. (2015). Rotinas de diagnósticos e tratamento das doenças infecciosas e parasitárias.

Wang, C., Horby, P. W., Hayden, F. G., \& Gao, G. F. (2020). A novel coronavirus outbreak of global health concern. The Lancet, 395(10223), 470-473. https://doi.org/10.1016/S0140-6736(20)30185-9

Wang, H., Ding, Y., Li, X., Yang, L., Zhang, W., \& Kang, W. (2003). Fatal aspergillosis in a patient with SARS who was treated with corticosteroids. The New England Journal of Medicine, 349(5), 507-508. https://doi.org/10.1056/NEJM200307313490519

Zuo, T., Zhan, H., Zhang, F., Liu, Q., Tso, E. Y. K., Lui, G. C. Y., Chen, N., Li, A., Lu, W., Chan, F. K. L., Chan, P. K. S., \& Ng, S. C. (2020). Alterations in Fecal Fungal Microbiome of Patients With COVID-19 During Time of Hospitalization until Discharge. Gastroenterology, 159(4), 1302-1310.e.5. https://doi.org/10.1053/j.gastro.2020.06.048 